\title{
Development of Thread Rolled Anti-Loosening Bolts Based on the Double Thread Mechanism and a Performance Evaluation*
}

\author{
Teruie TAKEMASU** and Hiroshi MIYAHARA**
}

\begin{abstract}
It has already been proven that bolt fasteners based on the double thread mechanism have an excellent anti-loosening performance. The purpose of this study is to establish a mass production method for these double thread bolts (DTBs) by thread rolling. The pitch ratio of the coarse thread and the fine thread of the target DTB is set as 2 to 1 . A two-die roller with a plunge feed is employed as the rolling method due to its fine processing precision. The roller dies used in the experiments have special grooves on the external surface which follow the same outline as the thread profiles of the DTB. Using these special dies, the DTB can be successfully formed in the same process as single thread bolts. The deformation of a workpiece during rolling is examined, and the examination shows that the formed material smoothly fills the die grooves in each cross section. The rolled DTBs completely pass the loosening test with extremely severe vibration and impact, as specified in NAS3354. The tensile fatigue strength of the rolled DTB is about $100 \%$ greater than that of the cutting DTB.
\end{abstract}

Key Words: Thread Rolling, Bolt, Double Thread Mechanism, Die Design, Loosening, Fatigue Strength

\section{Introduction}

Screw fasteners are definitely one of the most vital and useful parts of machinery. They have been used for a very long time in the West world, and they have currently spread almost worldwide. However, cyclic stresses during service can cause loosening and fatigue failure. It is well known that many accidents are caused by the loosening and fatigue failure of screw fasteners. Therefore, many researchers have investigated the causes of loosening of screw fasteners ${ }^{(1)-(4)}$ and they have considered many kinds of countermeasures ${ }^{(5)-(7)}$. But, most of the so-called anti-loosening bolts available in today's market cannot completely prevent the loosening.

It has been proven that bolt fasteners based on the double thread mechanism, as shown in Fig. 1, have an excellent anti-loosening ability due to their mechanical lock effect ${ }^{(8)}$. But, the double thread bolts (called DTBs hereafter) are currently manufactured by cutting due to the complicated structure of the threads. Thus, because DTBs are very expensive compared with commercial sin-

* Received 2nd June, 2005 (No. 05-4135)

** Department of Intelligent Machinery and Systems, Kyushu University, 744 Motooka, Nishi-ku, Fukuoka 8190395, Japan.

E-mail: gmzkckk@mech.kyushu-u.ac.jp gle thread bolts, their use has not been widespread in spite of their high market value.

Considering this background, the authors have developed a mass production method for DTBs by thread rolling, which is the production method for commercial single thread bolts. In this paper, we describe the prototype process, the processing results including the deformation, and we summarize the performance evaluations of the rolled DTB.

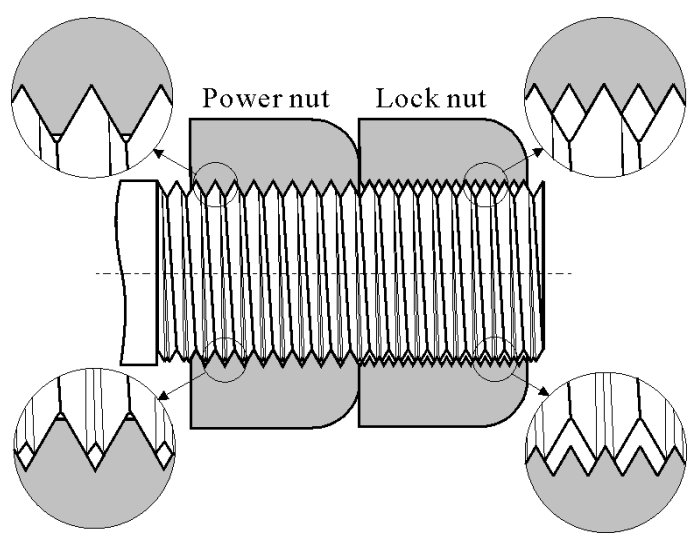

Fig. 1 Sketch of the DTB 


\section{Methods}

\subsection{Structure of the DTB}

Figure 1 shows the basic threaded structure of the DTB. As shown in this figure, the fine thread is coaxially superimposed over the coarse thread. The instructions for using the DTB are to add a power nut with a coarse pitch thread first, then to add a lock nut with a fine pitch thread to form the double nut structure. The double nut structure in the case of a single thread bolt prevents the screw fastener from loosening only by the friction acting on the contact surface between the two nuts. In contrast, the anti-loosening performance of the DTB is remarkably strengthened by the mechanical lock mechanism, that is, the interference effect of the two kinds of nuts. This effect can be explained as follows. It is very difficult to prevent the loosening of screw fasteners caused by the loosening rotation of the nut. In the case of the DTB, since the loosening velocity of the lock nut is slower than that of the power nut when both nuts rotate simultaneously in the loosening direction, the movement of the power nut is completely suppressed by the existence of the lock nut.

The pitch ratio between the coarse thread and the fine thread of the DTB can be decided arbitrarily. When that ratio is set as $m$ to $n$ ( $m>n$, where $m$ and $n$ are integers), the thread profile changes periodically for every $n$ pitches of the coarse thread. In this paper, the DTB of M12 in JIS with the pitch ratio of 2 to 1 was selected as the target product, because it has a strong market demand and it is easy to evaluate the processing state. Figure 2 (a) and (b) show the typical thread profiles of the DTB rolled in this study. The phase of the fine thread completely coin-

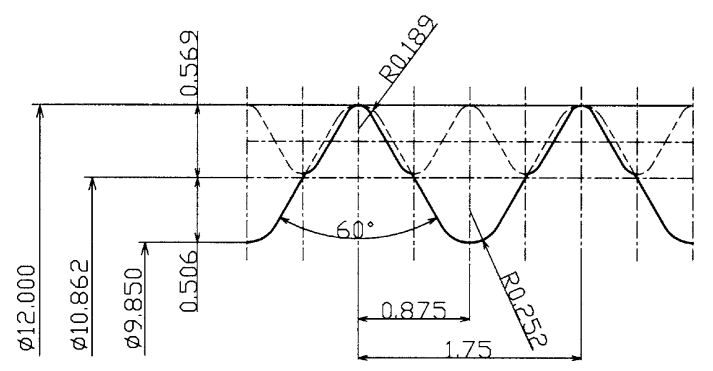

(a) 0 degree position

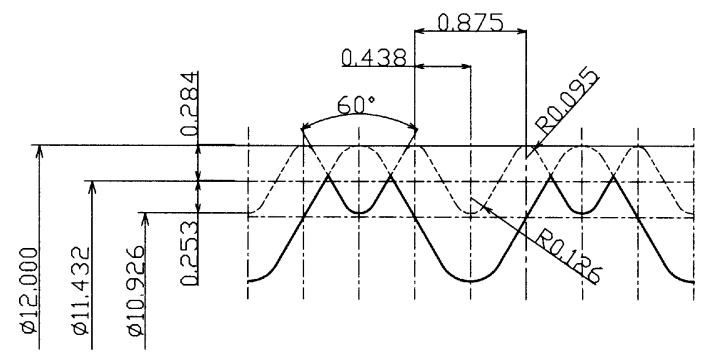

(b) 180 degree position

Fig. 2 Thread profiles of the DTB of M12 cides with the phase of the coarse thread at the 0-degree position, as shown in (a), and shifts from the phase of the coarse thread by just a half pitch of the fine thread at the 180-degree position, as shown in (b). Since the standard pitch of the coarse thread of M12 bolts is specified as $1.75 \mathrm{~mm}$ in JIS, the pitch of the fine thread is set at $0.875 \mathrm{~mm}$.

\subsection{Production method of the DTB}

In order to reduce the production costs, DTBs have to be manufactured by some mass production process, and thread rolling is undoubtedly the most suitable from the viewpoint of productivity and widespread use. In the thread rolling process of the DTB, both the coarse thread and the fine thread have to be formed simultaneously on the same rod axis. Although there seems to be several methods to realize this process, the authors employed the simplest way, which uses a special die (called the DTB die hereafter) in the single thread rolling process. This DTB die has grooves of a very complicated shape on the external surface, as shown in Fig. 3, which is the same outline as the thread profiles of the DTB in each corresponding cross section. These grooves can be formed only by a numerical controlled cylindrical grinding process.

\subsection{Experimental apparatus and conditions}

A two-die roller with a plunge feed was employed

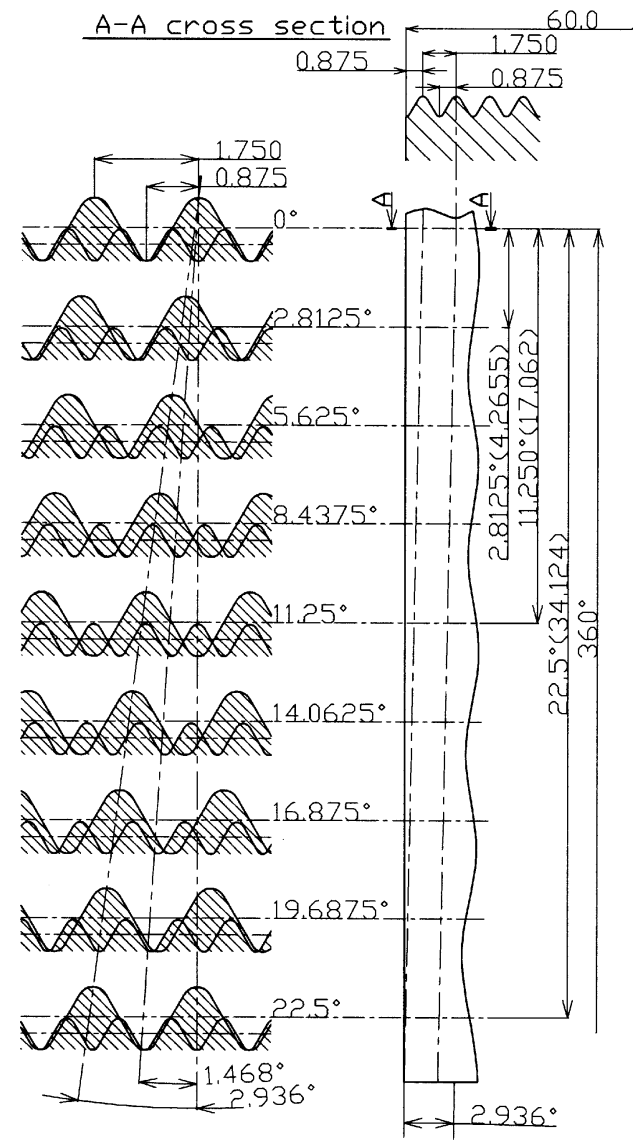

Fig. 3 Groove profiles of the rolling die for the DTB 


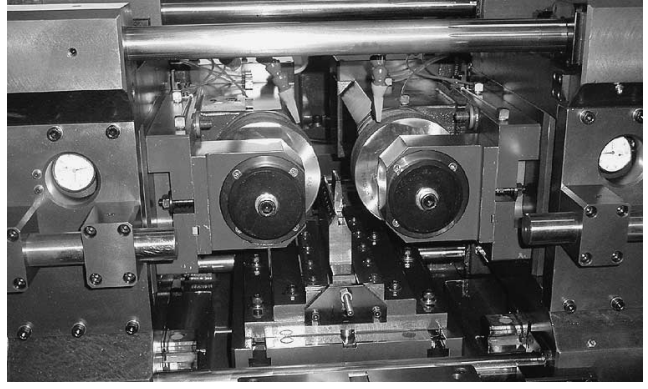

Fig. 4 Appearance of the experimental apparatus

as the rolling method due to its fine processing precision. Figure 4 shows the experimental apparatus. Two DTB roller dies are installed in each tool shaft, and a jig plate is set up between them to support the bolt material during rolling. The groove shape of the DTB die is very complicated and changes periodically according to the thread profiles. Therefore, the initial phase adjustment between the two DTB dies and the rotational synchronous accuracy during rolling is very important. The $\mathrm{CNC}$ form-rolling machine GA-160B, made by Nissei Co., Ltd., was used in this study. This apparatus has very high rigidity due to its four-poles structure. The tool shafts of this apparatus have a high-precision positioning mechanism which uses the numerical control feedback system. Therefore, the rotation angle error between both tool shafts can be reduced below 0.01 degree during rolling. The thread rolling conditions were as follows: the rotational speed of the tool was $60 \mathrm{rpm}$, the radial feed rate of the tool was $2 \mathrm{~mm} / \mathrm{sec}$ and the amount of tool indentation was $2.04 \mathrm{~mm}$. A machine structural steel, S45C in JIS, was selected as the bolt material, and the initial diameter was set at $10.75 \mathrm{~mm}$ after calculation from the volume constancy condition.

\section{Results and Discussion}

\subsection{Rolled DTB}

Figure 5 shows the appearance of the DTB rolled in this experiment. The shapes of the formed external threads based on the double thread mechanism were very fine and both the power nut and the lock nut could be added very easily. Figure 6(a) and (b) show the contact state of the DTB and the cross sections of the two kinds of nuts along the bolt axis at the 0 -degree position and the 180-degree position, respectively. The DTB threads fit very well with both the thread of the power nut and that of the lock nut. Additionally, no cutting chips or burrs were observed during rolling. Hence, these results indicate that the DTB can be successfully formed by the thread rolling process using the special dies proposed by the authors.

Figure 7 (a) and (b) show a zoomed comparison of the screw threads at the representative circumferential positions between the rolled DTB and the cutting DTB. The thread height and overall shape of the rolled DTB were al-

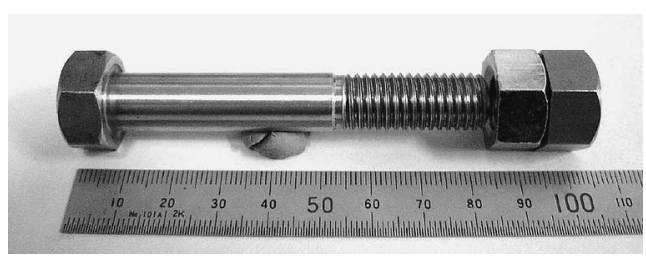

Fig. 5 Appearance of the rolled DTB
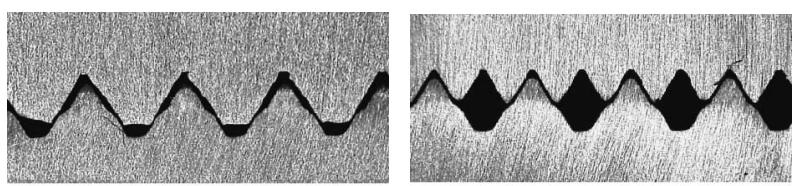

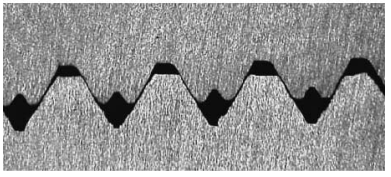

(a) Power nut

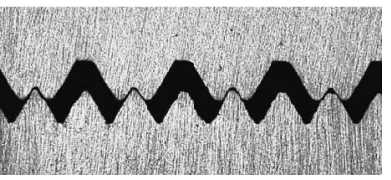

(b) Lock nut
Fig. 6 Contact state between the DTB and two kinds of nuts

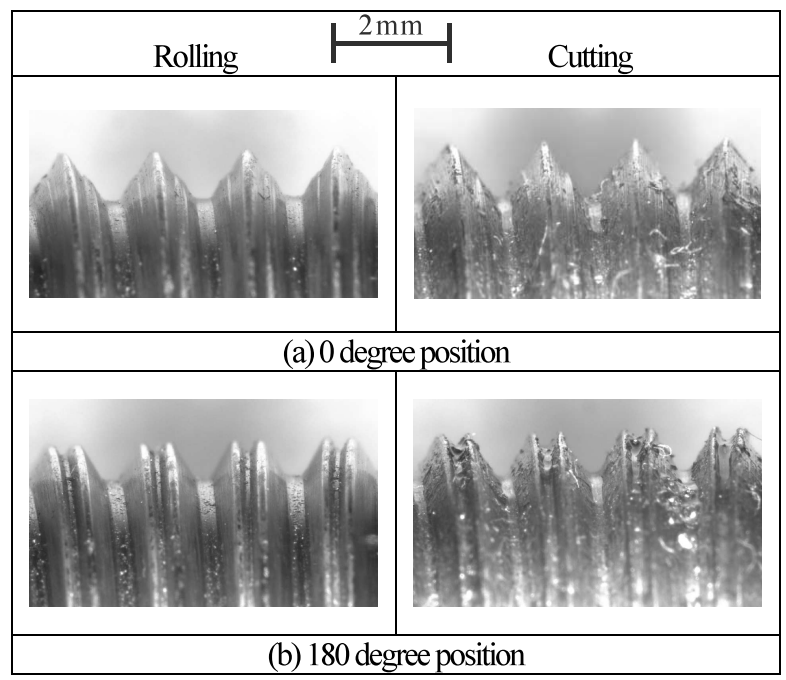

Fig. 7 Comparison of the thread appearance between the rolled DTB and the cutting DTB

most equivalent to those of the cutting DTB. The surface roughness of the cutting DTB was relatively rough due to burr formation during processing. On the other hand, the surface of the rolled DTB was very smooth due to the low surface roughness of the ground DTB dies, and the grooves of the fine screw thread were more clearly marked on the coarse screw thread.

\subsection{Thread rolling process of the DTB}

The material deformation behavior was observed in the amount of tool indentation. The tool indentation range of $0.62 \mathrm{~mm}-1.02 \mathrm{~mm}$ was divided into five stages by equal divisions of $0.1 \mathrm{~mm}$, and the stages were called stage- 1 through stage-5. Figure 8 shows the transition of the fill state of the material in each representative cross section of the DTB die grooves. Since only the coarse 


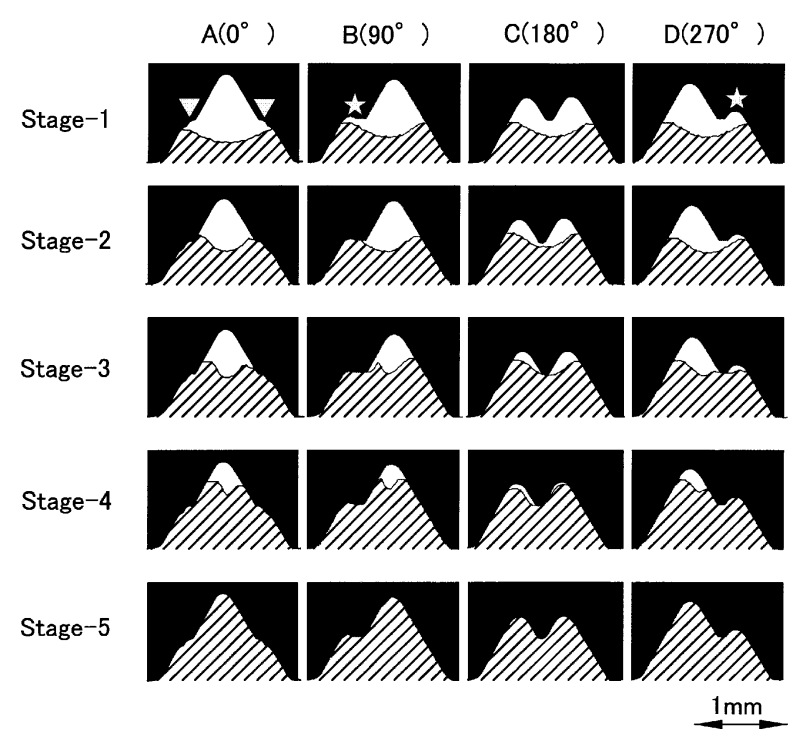

Fig. 8 Comparison of the fill state of material in each cross section of the die groove

thread part of the DTB was formed in stage-1, the deformation in every cross section at this stage was the same. The upper surface of the screw thread became concave and folding was observed in this stage.

Since the bottom shape of the die grooves changed periodically, the deformation manner and the fill state of the material were fairly different in each cross section after stage-1. In the A cross section (0-degree position), where the phase of the fine thread coincided with that of the coarse thread, the upper concave part gradually folded up and was finally crushed to fill the die groove. This was quite similar to the deformation pattern observed in the thread rolling process of single thread bolts. In the C cross section (180-degree position), where the phases of both threads were shifted by just a half pitch of the fine thread, the upper part of the folding moved up as a whole and the center of the bottom came in contact with the projection tip on the bottom of the die groove. Then, as the amount of the tool indentation increased, the material filled the gap of the die groove, as if it was wrapped up the projection. For this reason, in comparison with the other cross sections, the material flow in $\mathrm{C}$ was considered to be the smoothest. In the B cross section (90-degree position), the shallower left-side groove in the die bottom was first occupied by the piled-up edge of the thread upper surface. Then, the deeper-right side groove was filled in almost the same pattern as that observed in A. The deformation pattern in the $\mathrm{D}$ cross section (270-degree position) was almost the same as that observed in B.

Figure 9 (a) shows the relationship between the depth of the tool indentation and the amount of the fill area in each cross section, and (b) shows the fill ratio (the amount of fill area/groove area in each cross section). From stage1 to stage-4, the amount of the fill area is the maximum

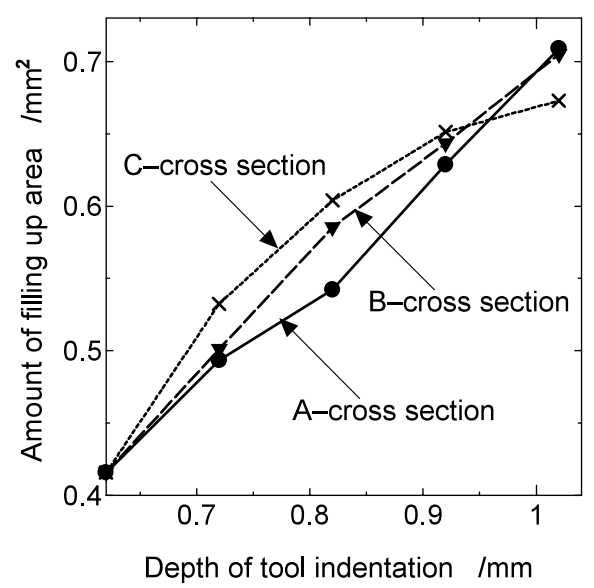

(a) Fill up area

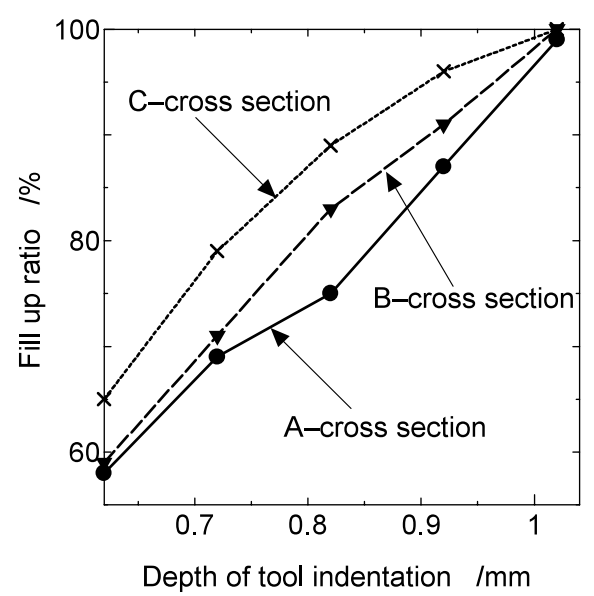

(b) Fill up ratio

Fig. 9 Relationship between the depth of tool indentation and the fill conditions of the die groove

in the $\mathrm{C}$ cross section, the minimum in $\mathrm{A}$, and in-between in $\mathrm{B}$. This is primarily due to the fact that there are steps, indicated by the marks of $\nabla$ and $\star$ in Fig. 8, which prevent the material from flowing into the bottom portion of these grooves. However, the fill rate of the material in $\mathrm{C}$ decreases gradually as the amount of the tool indentation increases. And the inclines of both curves in $\mathrm{C}$ become remarkably small when the amount of tool indentation exceeds about $0.9 \mathrm{~mm}$, since the amount of space becomes extremely smaller than those in the other cross sections at the end of stage-4. The final fill ratio in C reaches $100 \%$. The inclines of both characteristic curves in A decrease once in stage- 3 , then suddenly become steeper when the depth of tool indentation exceeds about $0.8 \mathrm{~mm}$, and the total amount of the fill area becomes the maximum at the end of stage-5. But, the final fill ratio reaches only about $98 \%$. The amount of the fill area in B increases almost linearly in proportion to the depth of the tool indentation, and the final fill ratio reaches almost $100 \%$. 


\section{Evaluation}

\section{1 Anti-loosening performance}

Comparative loosening tests by vibration were performed using the rolled DTB and the commercial single thread bolts. Figure 10 shows the vibration and impact testing device employed in this study. This device, specified in NAS3354, was designed to evaluate the effect of loosening due to vibration and an extremely severe impact force. As shown in this figure, a test bolt fastener with nuts, which is placed in the horizontal opening of the apparatus, is shaken up and down on the shaker stand. The frequency was $1780 \mathrm{cpm}$. The shaking stroke and the impact stroke were set to $11 \mathrm{~mm}$ and $19 \mathrm{~mm}$, respectively. An impact force of about $20 \mathrm{G}$ was applied by striking the workpiece at the upper and lower edges of the opening. A test was considered finished when the number of oscillations exceeded 30000 (about 17 minutes) or when the bolt fastener obviously loosened. After the loosening tests, the fastening torque was determined and each bolt fastener was examined for the existence of a crack or any other damage. Table 1 shows the results of the loosening tests for three kinds of fasteners. The commercial single thread bolts loosened in less than one minute, even in the case of the double nut structure. On the other hand, the DTB did not loosen during the regulated test time, and the fastening torque did not decrease at all. In addition, the DTB also passed an extended loosening test of about 10 hours

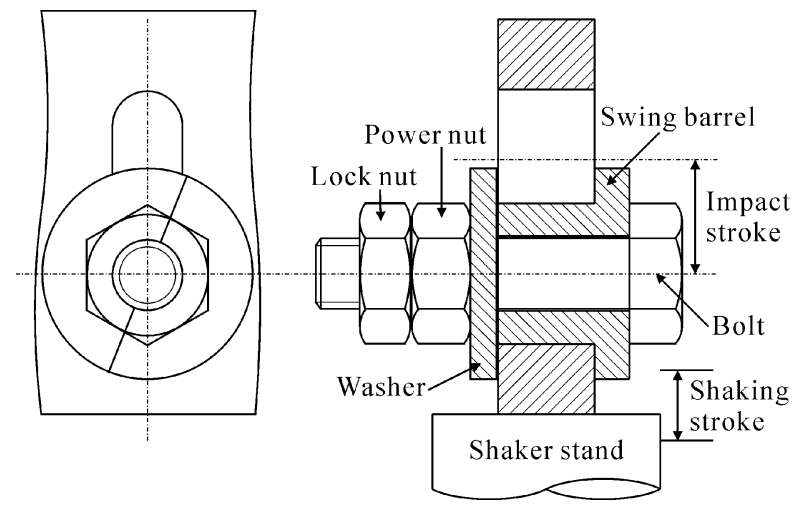

Fig. 10 Schema of the vibration and impact testing device

Table 1 Results of the vibration and impact test

\begin{tabular}{|c|c|c|c|}
\hline Specification & \multicolumn{2}{|c|}{ Fastening torque [Nm] } & \multirow{2}{*}{ Test results } \\
\cline { 2 - 3 } of screws & Power nut & Lock nut & \\
\hline $\begin{array}{c}\text { Single screw } \\
\text { bolt with } \\
\text { single nut }\end{array}$ & 50 & - & $\begin{array}{c}\text { Loosen for } \\
25 \text { seconds }\end{array}$ \\
\hline $\begin{array}{c}\text { Single screw } \\
\text { bolt with } \\
\text { double nuts }\end{array}$ & 50 & 50 & $\begin{array}{c}\text { Loosen for } \\
45 \text { seconds }\end{array}$ \\
\hline $\begin{array}{c}\text { Double screw } \\
\text { bolt with } \\
\text { double nuts }\end{array}$ & 50 & 30 & $\begin{array}{c}\text { Not loosen for } \\
17 \text { minutes }\end{array}$ \\
\hline
\end{tabular}

(the total number of oscillations was greater than $10^{7}$ ), and neither a crack nor any damage was observed. These results prove the excellent anti-loosening performance of the rolled DTB.

\subsection{Tensile fatigue strength}

Table 2 shows the four groups specimens used in the tensile fatigue tests. One group is the commercial single thread bolts, and other groups are the rolled DTBs and the cutting DTBs. All threads are of the M12 type, and the material is $\mathrm{S} 45 \mathrm{C}$. The tensile fatigue tests were conducted at room temperature using a standard 10-ton hydraulic servo pulser, EHM10, shown in Fig. 11. Sinusoidal wave loading with a mean stress of $200 \mathrm{MPa}$ and a frequency of $15 \mathrm{~Hz}$ was applied. The fatigue limit was assumed to be the maximum stress amplitude that endured stress cycles of $2 \times 10^{6}$.

Figure 12 shows the $S-N$ curves of the tensile fatigue tests. The fatigue limit of the commercial single thread bolts, group RS, is about $60 \mathrm{MPa}$, which is almost equal to the value described in the handbooks. The bolts of group CD (both threads are manufactured by cutting) withstood about $50 \mathrm{MPa}$. This value is less than the value obtained for RS, indicating that the fatigue lifetime of $\mathrm{CD}$ was not improved compared with RS. On the other hand, it should be noted that the bolts from groups RCD and RD, with coarse threads manufactured by rolling, attained $100 \mathrm{MPa}$ or more. This value is about two times larger than that of $\mathrm{CD}$ and about $80 \%$ larger than that of RS. These behav-

Table 2 Processing conditions of specimens

\begin{tabular}{|c|c|c|}
\hline \multirow{2}{*}{$\begin{array}{c}\text { Specimens } \\
{[\mathrm{M} 12]}\end{array}$} & \multicolumn{2}{|c|}{ Processing conditions } \\
\cline { 2 - 3 } & First pitch & Second pitch \\
\hline \hline RS & Rolling & - \\
\hline CD & Cutting & Cutting \\
\hline RCD & Rolling & Cutting \\
\hline RD & Rolling & Rolling \\
\hline
\end{tabular}

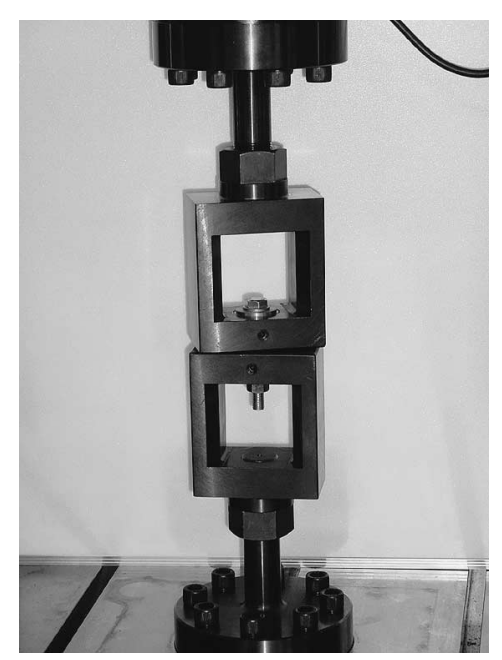

Fig. 11 Outer appearance of fatigue test 


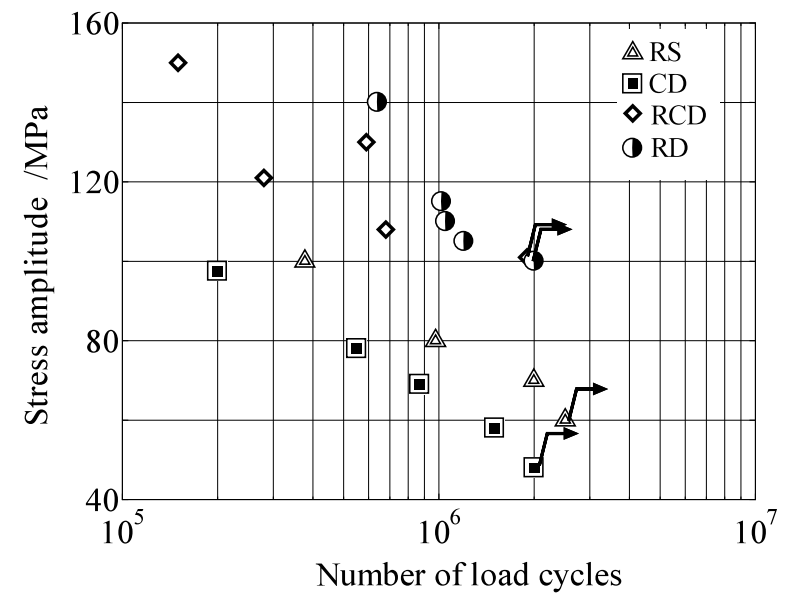

Fig. $12 S$ - $N$ curves

iors are primarily due to the anti-loosening effect of the DTB. In addition, cold rolling of the coarse thread causes the material to deform plastically, spread laterally and generate a work-hardening area and compressive residual stresses at the thread root. The combination of these factors is expected to lead to a significantly improved fatigue lifetime in the rolled DTBs.

\section{Conclusion}

(1) DTB can be successfully formed by the thread rolling using special dies proposed by the authors.

(2) In comparison with the cutting DTB, the surface finish of the rolled DTB was smoother. The grooves of the fine screw thread of the rolled DTB were more clearly marked on the coarse screw thread.

(3) The formed material smoothly filled the die grooves in each cross section.

(4) The rolled DTB did not loosen, even after the extended loosening test specified in NAS3354. Neither a crack nor any damage was observed.

( 5 ) The tensile fatigue strength of the rolled DTB increased to $100 \%$ greater than that of the cutting DTB.

\section{References}

( 1 ) Hongo, K., Loosening of Bolt and Nut Fastenings, Trans. Jpn. Soc. Mech. Eng., (in Japanese), Vol.30, No.215 (1964), pp.934-939.

( 2 ) Junker, G.H., New Criteria for Self-Loosening of Fasteners under Vibration, SAE Trans., (1969), pp.314335.

( 3 ) Yamamoto, A. and Kasei, S., Investigation of the SelfLoosening of Threaded Fasteners under Transverse Vibration, J. Precision Eng., (in Japanese), Vol.43, No.4 (1976), pp.470-475.

( 4 ) Jingu, T., Relationship between Loosening Response of Bolt-Nut Fastening System and Stress Wave Modes along the Direction of Propagation, Trans. Jpn. Soc. Mech. Eng., (in Japanese), Vol.61, No.586, A (1995), pp.1398-1403.

( 5 ) Sase, N., Nishioka, K., Koga, S. and Fujii, H., An AntiLoosening Screw-Fastener Innovation and Its Evaluation, J. Mater. Proc. Tech., 77 (1998), pp.209-215.

( 6 ) Jingu, T., Ishikawa, J. and Hatori, I., Protective Approach against Looseness of Screw Fastening Due to Impingement of Stress Wave, Trans. Jpn. Soc. Mech. Eng., (in Japanese), Vol.66, No.650, C (2000), pp.3475-3482.

( 7 ) Sase, N. and Fujii, H., Optimizing Study of SLBs for Higher Anti-Loosening Performance, J. Mater. Proc. Tech., 119 (2001), pp.174-179.

( 8 ) Nagawa, M., Noda, N., Ichihara, H., Yamada, M. and Saito, K., Study on Anti-Loosening Super Lock Nuts and Super Lock Bolts (First Volume), Machine Design, Vol.47, No.8 (2003), pp.35-42. 